\title{
Effect of High Fat Cholesterol Diet on Cerebrovascular Circulation and the Heart in Stroke-Prone SHR
}

\author{
Yukio Yamori, ${ }^{\circ *}$ Ryoichi Horie, ${ }^{\circ * *}$ Michiya Ohtaka, ${ }^{\circ * * *}$ \\ and Yasuo NARA ${ }^{\circ *}$
}

As we previously reported, stroke-prone SHR (SHRSP) show rather low serum cholesterol levels. We also clarified that a high fat cholesterol diet ( $\mathrm{HFC}$ diet) lowers the incidence of stroke in SHRSP (Yamori et al: Jap Heart J 17: 396, 1976). Previously confirmed was that moderate control of systemic blood pressure had a prophylactic effect on stroke in SHRSP (Yamori and Horie: Jap Circulat J 39: $616,1975)$. Therefore, from both macro- and microscopical findings and results of pressor responses to norepinephrine, we concluded that fatty degeneration in the media of the vascular wall probably decreased the functional response of blood vessels. Such degeneration also limited the increase in systemic blood pressure, so that the incidence of cerebrovascular lesions was clearly decreased. On the other hand, we first clarified at our laboratory that functional reduction of regional cerebral blood flow (rCBF) caused by severe hypertension precedes the occurrence of cerebrovascular organic damages (Yamori et al: Jap Jeart J 17:378, 1976). In the present studies, we examined $\mathrm{rCBF}$ and cerebrovascular reactivity in SHRSP on an HFC diet. We could already detect some characteristic electrocardiographical and/or vectorcardiographical findings in SHR in which macroscopical or microscopical left ventricular hypertrophy or myocardial infarction were confirmed at autopsy. Thus we examined the effect of HFC diet on the heart using electrocardiography (ECG) or vectorcardiography (VCG).

\section{Materials and Methods:}

Forty SHRSP and 20 WK from the age of 60 days were fed on an HFC diet of $20 \%$ suet, $5 \%$ cholesterol, and $2 \%$ cholic acid. Control groups for these were fed on normal laboratory stock diet (F II of Funahashi Co). These rats were repeatedly checked for blood pressure, body weight, $\mathrm{rCBF}$ (by the hydrogen clearance method), cerebrovascular reactivity in response to $10 \% \mathrm{CO}_{2}$ inhalation, and ECG or VCG.

Under pentobarbital anesthesia $(40 \mathrm{mg} / \mathrm{Kg}$ i.p.) rats were placed in a stereotaxic apparatus and enamel insulated platinum electrodes $(0.3 \mathrm{~mm}$ in diameter and $25 \mathrm{~mm}$ in length) were bilaterally implanted into the frontal regions, $2 \mathrm{~mm}$ apart from midsagittal line, $2 \mathrm{~mm}$ anterior to the bregma and $2.5 \mathrm{~mm}$ in depth from the cortical surface. These electrodes and a reference electrode on the mid-sagittal line, $4 \mathrm{~mm}$ arterior to the bregma, were connected to a miniature receptacle and the whole assembly was fixed on the skull with dental cement. rCBF was measured in conscious rats put in a small gas chamber, by Fujitani et al's modification of the hydrogen clearance method (Jap J Pharmacol 69: 328, 1973). Chemical cerebrovascular reactivities were detected, indicating an increase in $\mathrm{rCBF}$ response to

From the Japan Stroke Prevention Center (JSPC), "Izumo; Departments of Pathology* and Neurosurgery, ${ }^{* *}$ Faculty of Medicine, Kyoto University, Kyoto; the Center for Adult Diseases, *** Osaka. 
$10 \% \mathrm{CO}_{2}$ inhalation.

Before applying ECG and VCG to these rats, in order to get a close attachment of the electrode (usual metalic plates for ECG and large metalic ones especially made, $3 \times 4.5$ square $\mathrm{cm}$ for the frontal, $2 \times 2$ square $\mathrm{cm}$ for the other vectorcardiographical leads to the skin, the hair of these rats was neatly clipped, and a cotton mat the same size as the plate soaked in saline solution was placed between the skin and the plate. Under anesthesia (Nembutal, $40 \mathrm{mg} / \mathrm{Kg}$, i.p.) rats were fixed in prone position to the board and lead plates around the trunk were connected to an apparatus for ECG or VCG recording (Nihonkohden S-3013). Employed for ECG were ordinal extremity leads and 3 or 4 chest leads and for VCG the Takayasu lead system (6 leads consisting of 2 small electrodes and 4 large ones placed at the front, back, right, and left sides of the trunk) (Yamori et al: Jap Circulat J 40:1315, 1976).

\section{Results:}

As shown in the table, SHRSP fed on HFC diet showed moderate hypertension similar to that in stroke-resistant SHR(SHRSR), and showed no more rCBF decrease than SHRSR. In contrast, control SHRSP fed on normal diet developed severe hypertension over $200 \mathrm{mmHg}$, and showed marked decrease in rCBF. With regard to chemical cerebrovascular reactivity, it was revealed that SHRSP fed on HFC diet showed markedly reduced response to $\mathrm{CO}_{2}$ inhalation even in young age.

On the other hand, electrocardiographic and/or vectorcardiographic patterns in SHR with left ventricular hypertrophy and/or myocardial infarction were detected as follows:

With regard to left ventricular hypertrophy, left axis deviation, high voltage $\mathrm{R}$ waves in left chest leads and ST-T changes were observed electrocardiographically. Increased spatial QRS vector, left superior orientation of major QRS portion (left and posterior orientation usually observed in humans), and changes in ST-T vector were detected in VCG in SHR, just as in humans.

As for myocardial infarction, electro- and vectorcardiographical manifestation in SHR, e.g., abnormal $Q$ waves, ST-T changes, and ST-T vector changes well coincided with those in humans. However, in SHR the characteristic deviation and deformity of QRS vectors still remain unsolved, while in humans, such lesion specific deviation and deformity have been noted.

However, for the HFC diet mentioned above, effect on the heart was not so significant. That is, although patterns of myocardial organic changes were ob-

Table I. Effect of High Fat Cholesterol Diet (HFC Diet) on rCBF and Cerebrovascular Reactivity ( $A_{1}-$ sb Strain)

\begin{tabular}{|c|c|c|c|}
\hline & $\mathrm{HEC}$ & + & - \\
\hline \multicolumn{2}{|c|}{ Blood pressure (mmHg, $\mathrm{M}+\mathrm{SE}$ ) } & $178 \pm 2^{* *}$ & $210 \pm 9$ \\
\hline \multicolumn{2}{|c|}{$\mathrm{rCBF}$ in the Frontal Cortex $(\mathrm{M} \pm \mathrm{SE})$} & $102.6 \pm 11.6^{* *}$ & $65.6 \pm 6.3$ \\
\hline \multirow{2}{*}{$\begin{array}{l}\mathrm{rCBF} \text { Increase in } \\
\mathrm{Response} \text { to } \\
\mathrm{CO}_{2} \text { Inhalation }\end{array}$} & $(\mathrm{ml} / \mathrm{min} / 100 \mathrm{Gm}, \mathrm{M} \pm \mathrm{SE}$ & $129.3 \pm 13.0^{*}$ & $219.8 \pm 32.6$ \\
\hline & $(\%, \mathrm{M} \pm \mathrm{SE})$ & $240.5 \pm 21.7 *$ & $370.9 \pm 39.8$ \\
\hline
\end{tabular}

*, ** : statistically significant differences from control group $(\mathrm{p}<0.05,0.01)$ 
served in either ECG or VCG recording, statistical analysis of the precise components of waves in EGG and/or VCG revealed no remarkable differences between SHRSP fed on HFC diet and control SHRSP fed on normal diet.

Summary with Discussion:

The effect of high fat cholesterol diet (HFG diet) on cerebrovascular circulation was significant. I.e., at least at an early stage this diet lowered systemic blood pressure, thus leading to a reduced incidence of "arterionecro-thrombogenic " stroke by maintaining $\mathrm{rCBF}$ in normal range. Such result occurs despite clear ring-like fat deposition in the cerebrovascular system (Yamori et al: Stroke 7: 120, 1976), even though chemical cerebrovascular reactivity is reduced. This reduction of chemical cerebrovascular reactivity clearly indicates that fat deposits in cerebral arteries accelerate organic changes of cerebral arteries, i.e., cerebral arterio- or atherosclerosis.

The above-mentioned HFC diet showed little significance in relation to the heart, either electrocardiographically and/or vectorcardiographically. This coincides with our previous report that the degree of myocardial fibrosis was not so remarkable as compared with the control groups. However, problems involving contents of the HFG diet, strain differences in SHR, and duration of feeding remained to be solved in the near future.

This study was supported by the Science and Technology Agency of Japanese Government, Ministry of Education, National Institutes of Health, USA (Grant HL 17754), Japan Tabacco and Salt Public Cooperation, and Japan Heart Foundation. 